\title{
Single facility location problems with unbounded unit balls
}

\author{
Y. Hinojosa ${ }^{1}$, J. Puerto ${ }^{2}$ \\ ${ }^{1}$ Dept. Economía Aplicada I. Fac. Ciencias Económicas y E., Universidad de Sevilla, \\ 41018 Sevilla, Spain (e-mail: yhinojos@us.es) \\ ${ }^{2}$ Dept. Estadística e I.O., Facultad de Matemáticas, Universidad de Sevilla, \\ 41012 Sevilla, Spain (e-mail: puerto@us.es)
}

Manuscript received: August 2001/Final version received: January 2003

\begin{abstract}
In this paper we consider a new class of continuous location problems where the "distances" are measured by gauges of closed (not necessarily bounded) convex sets. These distance functions do not satisfy the definiteness property and therefore they can be used to model those situations where there exist zero-distance regions. We prove a geometrical characterization of these measures of distance as the length of shortest paths between points using only a subset of directions of their unit balls. We also characterize the complete set of optimal solutions for this class of continuous single facility location problems and we give resolution methods to solve them. Our analysis allows to consider new models of location problems and generalizes previously known results.
\end{abstract}

Key words: Continuous location, Convex analysis

\section{Introduction}

Location models are among the important problems in Applied Mathematics and Operations Research. They appear in different areas such as industrial engineering, systems analysis, computer science, transportation planning, ... Typical situations within the scope of location analysis are to locate items, activities, warehouses, machines to optimize transportation cost, to locate a silicon junction on an electronic chip to minimize distance covered, to determine the position of emergency units to minimize response time, ... (See e.g. Drezner [2] for different applications.) Another important application is in mathematical statistics. If the existing facilities are drawn according to a probability distribution, location analysis is used to search for good estimators minimizing different error measures (see e.g. Flury [8]). 
Many different elements may affect the formulation of a location problem: number of facilities to be located, space where the problem is considered (discrete, continuous, network, ...), type of objective function, nature of the service provided (attractive or repulsive), characteristics of the existing facilities, ... (See Hamacher and Nickel [9] for a classification of Location Problems and Drezner and Hamacher [3] for an up to date collection of papers on location analysis.)

Up to now most of the references in the literature concerning continuous location problems have considered distances induced by norms (see e.g. Drezner [2] or Drezner and Hamacher [3]). In the last years, there are also a number of papers that consider the use of gauges defined by the Minkowski functional of a compact convex set (not necessarily symmetric) containing the origin in its interior (see e.g. Durier and Michelot [4]; Fliege [7]; or RodriguezChia et al. [18]). These functions have been used in Location Theory to model situations where the symmetry property of a norm does not make sense. In this paper, we want to analyze more general models where the definiteness property of the gauge of a compact convex set is relaxed. Relaxing definiteness introduces the existence of zero-distance regions. It is easy to see that a gauge of a closed convex set containing the origin has a zero-distance region (different from the zero-vector) if and only if its unit ball is unbounded. (Figure 1 shows several distance level curves with respect to some zerodistance regions.)
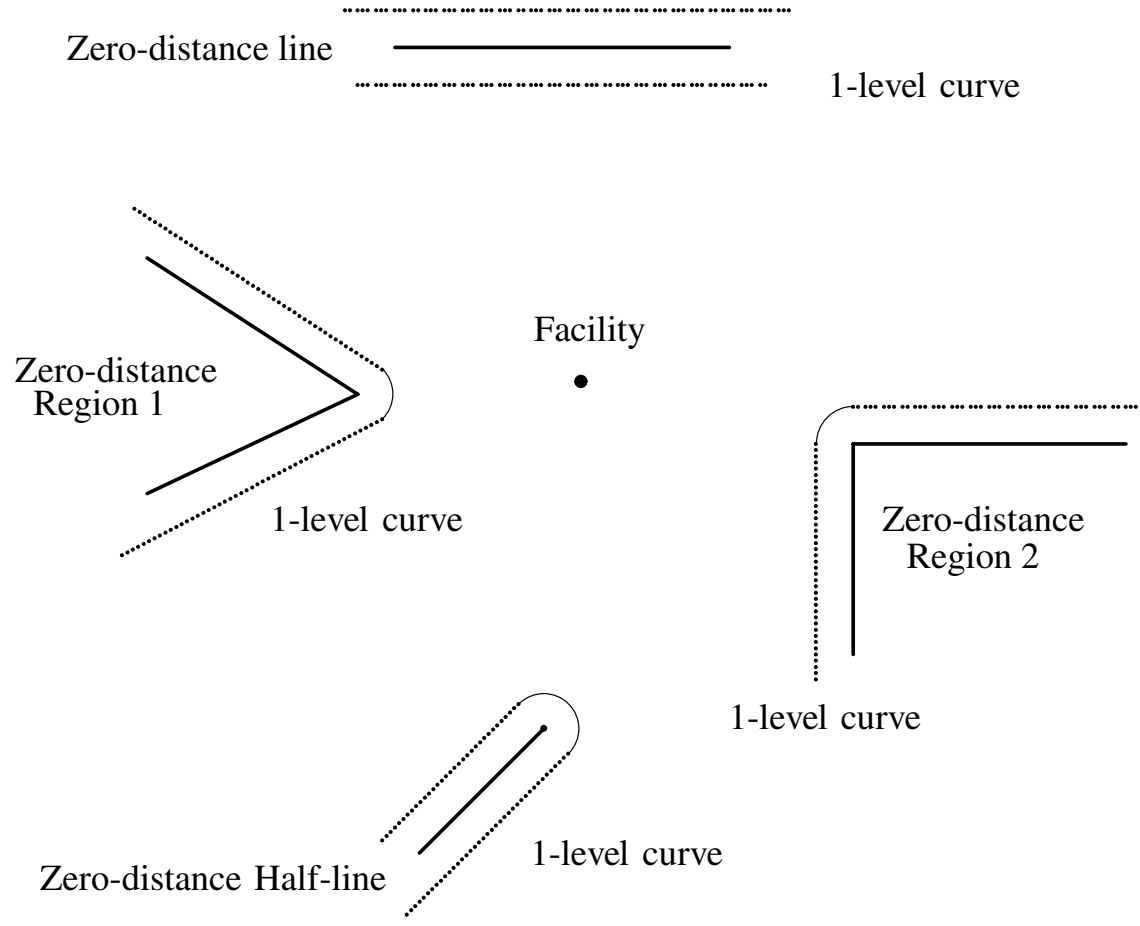

Fig. 1. Distance level curves with respect to zero-distance regions 
The simplest location problem with zero-distance regions that one can consider consists of locating a new facility minimizing the sum of the distances with respect to a given set of lines (see Robert and Toussaint [16]). This problem is the dual of a very well-known problem consisting of locating a line minimizing the distances to a given set of points (see Morris and Norback [14], [15]; Megiddo and Tamir [13]; Love, Morris and Wesolowsky [12]; Schöbel [19] or Robert and Toussaint [16]). After a first analysis, this problem reduces to a linear programming problem (see Subsection 4.1 for further details). Nevertheless, although this problem is easy to solve, the general model presents an interesting structure worth to be investigated.

Our aim in this paper is to analyze Location Problems in a general framework where gauges of closed (not necessarily bounded) convex sets are used to model distances. Gauges of compact convex sets have a very interesting property: The distance between two points is the shortest path between them using only fundamental directions of the unit ball. First, we prove that this geometrical interpretation can be extended to gauges of closed convex sets by considering trip directions of a specific convex subset of the unit ball. Second, we give a geometrical characterization of the set of optimal solutions for this family of problems.

It is worth noting that all the literature of location analysis based on gauges of compact convex sets can be seen as a particular case of this approach. Besides, new types of location problems can be considered and solved.

The paper is organized as follows. Section 2 is devoted to the mathematical preliminaries. That section also includes a proof of the geometrical interpretation of distances measured by gauges of closed convex sets. In Section 3 a general location problem with gauges of closed convex sets is introduced and those cases where the optimal objective value is either 0 or unbounded are discussed. Section 4 deals with the geometrical characterization of the set of optimal solutions. Two particularly interesting cases are included: the Weber problem and the minimax problem. The last section is devoted to some concluding remarks.

\section{Mathematical preliminaries}

In this paper everything takes place in the Euclidean space $\mathbb{R}^{n}$ where the inner product is denoted by $\langle\cdot, \cdot\rangle$. We will consider gauges of closed (not necessarily bounded) convex sets as measures of distance. Thus, in this section we recall the concept of gauge and we relate it to some other concepts of convex analysis. (Further details can be found in Hiriart-Urruty and Lemaréchal [10], or Rockafellar [17].) by

Let $C$ be a closed convex set containing the origin. The function $\varphi$ defined

$$
\varphi(x):=\inf \{\alpha>0: x \in \alpha C\}
$$

is called the gauge of $C$. The set $C$ will be called the unit ball associated with $\varphi$. For convenience, we set $\varphi(x):=+\infty$ if $x \in \alpha C$ for no $\alpha>0$.

The expression (1) is the definition of gauge used in convex analysis (see Definition V.1.2.4 in Hiriart-Urruty and Lemaréchal [10]). Nevertheless, the term gauge has been used in Location Theory (since the seminal paper by 
Witzgall [22]) to denote the functional $\varphi$ associated to a compact convex set containing the origin in its interior. In this paper, we use the term gauge in its full dimension: it denotes the functional $\varphi$ associated to a closed (not necessarily bounded) convex set containing the origin (not necessarily in its interior).

Notice that the gauge function $\varphi$ is finite if and only if the zero vector lies in the interior of its unit ball $C$. If zero lies on the boundary of $C$, the set of points where $\varphi(x)=+\infty$ is the complementary set of the conical hull of $C, \mathbb{R}^{n} \backslash$ cone $(C)$. On the other hand, it is easy to see that a gauge function $\varphi$ verifies the definiteness property if and only if its unit ball is bounded. Thus, in general the definiteness property is not satisfied by gauges of closed convex sets. This remark leads us to recall the concept of asymptotic cone.

Let $\varphi$ be the gauge of a closed convex set $C$ containing the origin, the asymptotic cone of $\varphi$ is given by

$$
C_{\infty}:=\left\{x \in \mathbb{R}^{n}: \varphi(x)=0\right\} .
$$

It is worth nothing that $C_{\infty}$ is a closed convex cone determined by the directions of recession of $C$ (see pages 108 and 203 in Hiriart-Urruty and Lemaréchal Part I [10]) i.e.,

$$
C_{\infty}=\left\{d \in \mathbb{R}^{n}: x+\lambda d \in C \quad \forall x \in C, \quad \forall \lambda>0\right\} .
$$

Let $\varphi$ be the gauge of a closed convex set $C$ containing the origin. We define the "distance" from $y$ to $x$ by $\varphi(x-y)$. This "distance" function verifies the following properties:

1. $\varphi(x-y) \geq 0 \quad \forall x, y \in \mathbb{R}^{n}$.

2. $\varphi(x-y)=0 \Longleftrightarrow x-y \in C_{\infty}$.

3. $\varphi(x-y) \leq \varphi(x-z)+\varphi(z-y) \quad \forall x, y, z \in \mathbb{R}^{n}$.

Notice that if the unit ball $C$ is bounded, $C_{\infty}=\{\boldsymbol{0}\}$ and thus, $\varphi(x-y)=0$ if and only if $x=y$. If in addition $C$ is symmetric with respect to the origin, $\varphi$ is a norm and we have the additional property $\varphi(x-y)=\varphi(y-x)$.

For the sake of readability we show several examples of gauges with unbounded unit ball (see Figure 2).

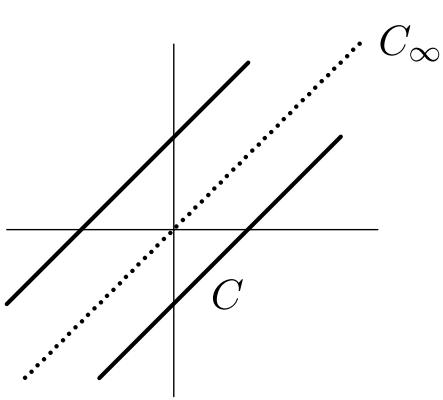

Quadratic gauge

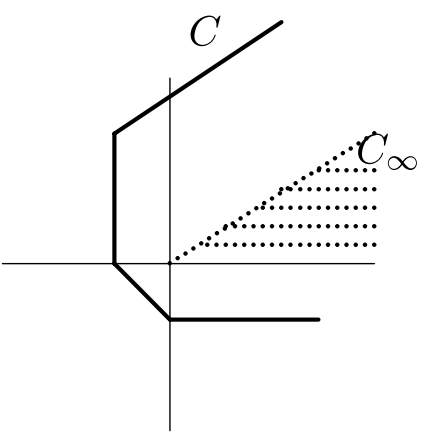

Polyhedral gauge

Fig. 2. Examples of gauges with unbounded unit ball $C$ 
Example 1. Quadratic gauges. Let $Q$ be a symmetric positive semi-definite matrix in $\mathbb{R}^{n \times n}$. A quadratic gauge is defined by

$$
\varphi(x):=\sqrt{\langle Q x, x\rangle} \text { for all } x \in \mathbb{R}^{n} .
$$

If $Q \in \mathbb{R}^{2 \times 2}, Q=\left(\begin{array}{ll}q_{11} & q_{12} \\ q_{12} & q_{22}\end{array}\right)$ with $q_{11}>0, q_{22}>0$, and $q_{11} q_{22}=q_{12}^{2}$; then $\varphi\left(x_{1}, x_{2}\right)=\left|\frac{q_{11}}{\sqrt{q_{11}}} x_{1}+\frac{q_{12}}{\sqrt{q_{11}}} x_{2}\right|$. The unit ball is given by $C=\left\{\left(x_{1}, x_{2}\right) \in \mathbb{R}^{2}\right.$ : $\left.\left|\frac{q_{11}}{\sqrt{q_{11}}} x_{1}+\frac{q_{12}}{\sqrt{q_{11}}} x_{2}\right| \leq 1\right\}$; and the asymptotic cone is $C_{\infty}=\left\{\left(x_{1}, x_{2}\right) \in \mathbb{R}^{2}\right.$ : $\left.x_{2}=-\frac{q_{11}}{q_{12}} x_{1}\right\}$.

Notice that if $\varphi$ is a quadratic gauge in $\mathbb{R}^{2}$, its unit ball $C$ is a symmetric strip and its asymptotic cone $C_{\infty}$ is a line passing through the origin.

Example 2. Polyhedral gauges. Let $s_{i} \in \mathbb{R}^{n}$ for $i=1, \ldots, p$ and let $C=\left\{x \in \mathbb{R}^{n}:\left\langle s_{i}, x\right\rangle \leq b_{i}, \quad b_{i} \geq 0 \quad \forall i=1, \ldots, p\right\}$ be a closed convex polyhedron containing the origin. The function $\varphi$ defined by (1) is called a polyhedral gauge. The asymptotic cone of $\varphi$ is a closed convex cone given by $C_{\infty}=\left\{x \in \mathbb{R}^{n}:\left\langle s_{i}, x\right\rangle \leq 0 \quad \forall i=1, \ldots, p\right\}$.

Once the concept of gauge of a closed convex set has been introduced the next step is to characterize how to evaluate it. There are two alternative ways to evaluate a gauge. The first one is by means of the polar set of its unit ball.

Let $C$ be a closed convex set containing the origin. The polar set of $C$ is given by

$$
C^{*}=\left\{s \in \mathbb{R}^{n}:\langle s, c\rangle \leq 1 \quad \forall c \in C\right\} .
$$

It is well-know that the gauge of a closed convex set $C$ containing the origin is the support function of the polar set $C^{*}$ (see Theorem 14.5 in Rockafellar [17]). Therefore,

$$
\varphi(x)=\sup _{c \in C^{*}}\langle c, x\rangle .
$$

The second alternative to evaluate the gauge $\varphi$ of a closed convex set containing the origin gives us a geometrical interpretation of this value as the length of a particular shortest path. We show that $\varphi(x)$ is the shortest path from the origin to the point $x$ using only trip directions of a subset of points of the gauge unit ball.

In what follows we denote by $\operatorname{ext}(C),(C)_{\infty}^{e x t}, \operatorname{conv}(C)$, and $\operatorname{cone}(C)$, the set of extreme points, the set of extreme directions, the convex hull, and the conical hull of the set $C$, respectively. By $L^{\perp}$ is denoted the orthogonal subspace to a linear subspace $L$.

Definition 1. A convex set $C$ has linearity zero if it contains no lines.

Lemma 1. Let $C$ be a closed convex set with non-zero linearity, let $L$ be the linearity subspace of $C$, and let $C_{0}=C \cap L^{\perp}$. Then, the set $C$ can be expressed as

$$
C=\operatorname{conv}\left(\operatorname{ext}\left(C_{0}\right)\right)+\operatorname{cone}\left(\left(C_{0}\right)_{\infty}^{e x t}\right)+L .
$$

Notice that $C_{0}$ is a closed convex set with zero linearity, and that $C=C_{0}+L$. Thus, the proof is a direct consequence of Theorem 18.5 in Rockafellar [17].

The following theorem gives us an interpretation of the gauge of a closed convex set $C$ as the shortest path between points using only displacement in the directions given by the extreme points of $C_{0}$. 
Theorem 1. Let $C$ be a closed convex set containing the origin, with linearity subspace $L$, and let $C_{0}=C \cap L^{\perp}$. Suppose the extreme points of $C_{0}$ are $\operatorname{ext}\left(C_{0}\right):=\left\{b_{i}\right\}_{i \in I}$, the extreme directions of $C_{0}$ are $\left(C_{0}\right)_{\infty}^{\text {ext }}:=\left\{d_{j}\right\}_{j \in J}$, and $\left\{v_{1}, \ldots, v_{p}\right\}$ is a basis of L. Then, for each point $x$ there exist $\left\{\lambda_{i}: \lambda_{i} \geq 0\right\}_{i \in I}$, $\left\{\mu_{j}: \mu_{j} \geq 0\right\}_{j \in J}$, and $\left\{\delta_{k}: \delta_{k} \in \mathbb{R}\right\}_{k=1}^{p}$ such that the gauge of $C$ at point $x$ given $b y$

$$
\varphi(x)=\inf \left\{\sum_{i \in I} \lambda_{i}: x=\sum_{i \in I} \lambda_{i} b_{i}+\sum_{j \in J} \mu_{j} d_{j}+\sum_{k=1}^{p} \delta_{k} v_{k}\right\} .
$$

Moreover, if $I_{0}(x):=\left\{i: \lambda_{i}>0\right\}$ and $J_{0}(x):=\left\{j: \mu_{j}>0\right\}$, then $\left|I_{0}(x)\right|+$ $\left|J_{0}(x)\right| \leq n+1$.

Proof. By Lemma 1 we know that for each $x \in C$ there exist $\left\{\lambda_{i}^{\prime}: \lambda_{i}^{\prime} \geq 0\right\}_{i \in I}$ verifying $\sum_{i \in I} \lambda_{i}^{\prime}=1,\left\{\mu_{j}^{\prime}: \mu_{j}^{\prime} \geq 0\right\}_{j \in J}$, and $\left\{\delta_{k}^{\prime}: \delta_{k}^{\prime} \in \mathbb{R}\right\}_{k=1}^{p}$ such that the point $x$ can be written as

$$
x=\sum_{i \in I} \lambda_{i}^{\prime} b_{i}+\sum_{j \in J} \mu_{j}^{\prime} d_{j}+\sum_{k=1}^{p} \delta_{k}^{\prime} v_{k} .
$$

In addition, by Caratheodory's Theorem we know that any point of $C_{0}$ can be expressed as a convex combination of at most $n+1$ extreme points and directions of $C_{0}$. Therefore, if $I_{0}(x):=\left\{i: \lambda_{i}^{\prime}>0\right\}$ and $J_{0}(x):=\left\{j: \mu_{j}^{\prime}>0\right\}$, then $\left|I_{0}(x)\right|+\left|J_{0}(x)\right| \leq n+1$.

On the other hand, we know from (1) that

$$
\varphi(x)=\inf \left\{\alpha>0: \frac{x}{\alpha} \in C\right\} \text {. }
$$

Combining both results we obtain that there exist $\left\{\lambda_{i}^{\prime}: \lambda_{i}^{\prime} \geq 0\right\}_{i \in I}$ verifying $\sum_{i \in I} \lambda_{i}^{\prime}=1,\left\{\mu_{j}^{\prime}: \mu_{j}^{\prime} \geq 0\right\}_{j \in J}$, and $\left\{\delta_{k}^{\prime}: \delta_{k}^{\prime} \in \mathbb{R}\right\}_{k=1}^{p}$ such that

$$
\varphi(x)=\inf \left\{\alpha>0: \frac{x}{\alpha}=\sum_{i \in I} \lambda_{i}^{\prime} b_{i}+\sum_{j \in J} \mu_{j}^{\prime} d_{j}+\sum_{k=1}^{p} \delta_{k}^{\prime} v_{k}\right\}
$$

and moreover, $\left|I_{0}\left(\frac{x}{\alpha}\right)\right|+\left|J_{0}\left(\frac{x}{\alpha}\right)\right| \leq n+1$. Take $\lambda_{i}=\alpha \lambda_{i}^{\prime}$ for all $i \in I$, then we have that $\sum_{i \in I} \lambda_{i}=\alpha$. Take in addition $\mu_{j}=\alpha \mu_{j}^{\prime}$ for all $j \in J$ and $\delta_{k}=\alpha \delta_{k}^{\prime}$ for all $k=$ $1, \ldots, p$, thus we obtain that for each $x$ there exist $\left\{\lambda_{i}: \lambda_{i} \geq 0\right\}_{i \in I},\left\{\mu_{j}: \mu_{j} \geq\right.$ $0\}_{j \in J}$, and $\left\{\delta_{k}: \delta_{k} \in \mathbb{R}\right\}_{k=1}^{p}$ such that the gauge of $C$ at point $x$ is given by

$$
\varphi(x)=\inf \left\{\sum_{i \in I} \lambda_{i}: x=\sum_{i \in I} \lambda_{i} b_{i}+\sum_{j \in J} \mu_{j} d_{j}+\sum_{k=1}^{p} \delta_{k} v_{k}\right\} .
$$

Moreover, $I_{0}(x)=I_{0}\left(\frac{x}{\alpha}\right), J_{0}(x)=J_{0}\left(\frac{x}{\alpha}\right)$ and thus $\left|I_{0}(x)\right|+\left|J_{0}(x)\right| \leq n+1$.

This characterization extends a previous result given by Ward and Wendell [20] stating that the block norm of a point $x$ is the length of one of the shortest paths from the origin to $x$ using only trip directions of the unit ball. In Theorem 1 we show that the same interpretation can be applied to a particular expansion of the point $x$ in terms of extreme points and directions of a specific convex subset of the unit ball and a basis of the linearity subspace of $C$. It is worth noting that if the set $C$ has zero linearity, the last term in the expansion of $x$ disappears. Nevertheless, this does not modify neither the interpretation nor the evaluation of $\varphi(x)$. 
Finally, we conclude this section including the expression of the subdifferential set of the gauge of a closed convex set. We will need this set in Section 4. (For more details see Hiriart-Urruty and Lemaréchal [10].)

Let $\partial \varphi(x)$ be the subdifferential set of the function $\varphi$ at point $x$, let $\varphi^{*}$ be the conjugate of the function $\varphi$, and let $N_{C}(x)$ be the normal cone to the set $C$ at point $x$. It is well-known that for any closed convex function $\varphi, s \in \partial \varphi(x)$ if and only if $x \in \partial \varphi^{*}(s)$ (see Corollary 1.4.4 in Hiriart-Urruty and Lemaréchal, Part II [10]). The subdifferential set of the conjugate function $\varphi^{*}$ is given by

$$
\partial \varphi^{*}(s)= \begin{cases}N_{C^{*}}(s) & \text { if } s \in C^{*} \\ \emptyset & \text { if } s \notin C^{*}\end{cases}
$$

Then, since $C^{*}$ is closed, for each $x$ such that $\varphi(x)<+\infty$ we have that

$$
\begin{aligned}
\partial \varphi(x) & =\left\{s \in C^{*}: x \in N_{C^{*}}(s)\right\} \\
& =\left\{s \in C^{*}:\langle x, s\rangle=\varphi(x)\right\} .
\end{aligned}
$$

Notice that if $x=0$, then $\partial \varphi(0)=C^{*}$ and if $x \in C_{\infty}$, then $0 \in \partial \varphi(x)$. Notice also that if $\varphi$ is a quadratic gauge, $C^{*}$ is orthogonal to $C_{\infty}$ and thus, $\partial \varphi(x)=C^{*}$ for all $x \in C_{\infty}$.

\section{Problem formulation}

Throughout this paper $A$ is a finite subset of $\mathbb{R}^{n}$, which represents the set of existing facilities. Each facility $a \in A$ has associated a gauge $\varphi_{a}$ whose unit ball is a closed convex set containing the origin. The distance from an existing facility $a \in A$ to a new facility $x \in \mathbb{R}^{n}$ is assumed to be given by $\varphi_{a}(x-a)$. We are looking for the location of a new facility $x \in \mathbb{R}^{n}$ that minimizes a globalizing function $\gamma$, which depends on the weighted distance from each existing facility to the new facility.

We are given a set of existing facilities $A:=\left\{a_{1}, \ldots, a_{m}\right\}$. Let $w=\left\{w_{a_{1}}, \ldots, w_{a_{m}}\right\}$ be a set of positive weights; and let $\gamma: \mathbb{R}^{m} \longrightarrow \mathbb{R}$ be a monotone gauge of a closed convex set containing the origin. Recall that a function $\gamma$ is a monotone gauge on $\mathbb{R}^{m}$ if $\gamma(u) \leq \gamma(v)$ for every $u$ and $v$ in $\mathbb{R}^{m}$ satisfying $\left|u_{i}\right| \leq\left|v_{i}\right|$ for each $i=1, \ldots, m$ (see Johnson and Nylen [11] for further details on monotone norms). The mathematical formulation of the problem is

$$
P_{w}^{\gamma}(A) \quad \min _{x \in \mathbb{R}^{n}} F(x)=\gamma\left(w_{a_{1}} \varphi_{a_{1}}\left(x-a_{1}\right), \ldots, w_{a_{m}} \varphi_{a_{m}}\left(x-a_{m}\right)\right) .
$$

We will denote by $F^{*}$ the optimal objective value of Problem $P_{w}^{\gamma}(A)$ and as it is usual, the set of minimizers of $P_{w}^{\gamma}(A)$ will be denoted by $M_{w}^{\gamma}(A)$.

The following remark shows that interesting, well-know problems in Location Theory are particular cases of this general location problem.

Remark 1. For $\gamma(x)=\sum_{i=1}^{m}\left|x_{i}\right|$ the problem becomes a generalization of the usual Weber problem (see e.g. Wesolowsky [21]). For $\gamma(x)=\max _{i}\left|x_{i}\right|$ we get a generalization of the center problem (see e.g. Elzinga and Hearn [6]). Finally, for $\gamma(x)=\lambda \sum_{i=1}^{m}\left|x_{i}\right|+(1-\lambda) \max _{i}\left|x_{i}\right|$ with $0<\lambda<1$ we have a generalization of the cent-dian problem (see e.g. Carrizosa et al. [1]).

One of the goals in this paper is to characterize the whole set of optimal solutions of Problem $P_{w}^{\gamma}(A)$. This problem has the novelty with respect to 
previous formulations in Location Theory that its optimal objective value may be 0 or unbounded. First of all, we characterize these extreme cases and next we proceed to the non-degenerate cases.

For every $a \in A$ we denote by $C_{a}$ the unit ball of $\varphi_{a}$, by $C_{a}^{*}$ the polar set of $C_{a}$ and by $\left(C_{a}\right)_{\infty}$ the asymptotic cone of $\varphi_{a}$. In the same way, we denote by $C_{\gamma}$ and by $\left(C_{\gamma}\right)_{\infty}$ the unit ball and the asymptotic cone of $\gamma$, respectively.

Lemma 2. Suppose that the globalizing function $\gamma$ is the gauge of a bounded closed convex set. Then, the optimal objective value of Problem $P_{w}^{\gamma}(A)$ is zero if and only if the following condition is fulfilled:

$$
S:=\bigcap_{i=1}^{m}\left(a_{i}+\left(C_{a_{i}}\right)_{\infty}\right) \neq \emptyset \text {. }
$$

In addition, in this case $M_{w}^{\gamma}(A)=S$.

Proof. If $S \neq \emptyset$, there exists $x^{*} \in \mathbb{R}^{n}$ such that $x^{*}-a_{i} \in\left(C_{a_{i}}\right)_{\infty} \forall i=1, \ldots, m$. Then, $\varphi_{a_{i}}\left(x^{*}-a_{i}\right)=0 \quad \forall i=1, \ldots, m$ and thus $F\left(x^{*}\right)=\gamma(\mathbf{0})=0$. Therefore, since $\gamma(x) \geq 0$ for all $x \in R^{n}$, the optimal objective value is zero and $S=M_{w}^{\gamma}(A)$.

Conversely, if $F^{*}=0$, there exists $x^{*} \in \mathbb{R}^{n}$ such that $F\left(x^{*}\right)=0$. Thus, since $\gamma$ is the gauge of a compact convex set, $w_{a_{i}} \varphi_{a_{i}}\left(x^{*}-a_{i}\right)=0 \forall i=1, \ldots, m$. Therefore, $x^{*}-a_{i} \in\left(C_{a_{i}}\right)_{\infty} \forall i=1, \ldots, m$ and the condition is fulfilled.

Lemma 3. Suppose that the globalizing function $\gamma$ is the gauge of an unbounded closed convex set. Then, the optimal objective value of Problem $P_{w}^{\gamma}(A)$ is zero if and only if the following condition is fulfilled:

$$
T:=\left\{x \in \mathbb{R}^{n}:\left(w_{a_{1}} \varphi_{a_{1}}\left(x-a_{1}\right), \ldots, w_{a_{m}} \varphi_{a_{m}}\left(x-a_{m}\right)\right) \in\left(C_{\gamma}\right)_{\infty}\right\} \neq \emptyset .
$$

In addition, in this case we have that $M_{w}^{\gamma}(A)=T$.

Proof. If $T \neq \emptyset$, it is straightforward to see that $F^{*}=0$ and that $M_{w}^{\gamma}(A)=T$.

Conversely, if $F^{*}=0$, there exists $x^{*} \in R^{n}$ such that $F\left(x^{*}\right)=0$. Thus, $\left(w_{a_{1}} \varphi_{a_{1}}\left(x^{*}-a_{1}\right), \ldots, w_{a_{m}} \varphi_{a_{m}}\left(x^{*}-a_{m}\right)\right) \in\left(C_{\gamma}\right)_{\infty}$ and the result follows.

Notice that $S \subseteq T$. Thus, if the globalizing function $\gamma$ is the gauge of an unbounded closed convex set, condition (11) is also a sufficient condition for $F^{*}=0$.

Lemma 4. Suppose that the globalizing function $\gamma$ is the gauge of a bounded closed convex set containing the origin in its interior. Then, the optimal objective value for Problem $P_{w}^{\gamma}(A)$ is unbounded if and only if

$$
\bigcap_{i=1}^{m}\left(a_{i}+\operatorname{cone}\left(C_{a_{i}}\right)\right)=\emptyset \text {. }
$$

Proof. If $\bigcap_{i=1}^{m}\left(a_{i}+\operatorname{cone}\left(C_{a_{i}}\right)\right)=\emptyset$, then for all $x \in \mathbb{R}^{n}$ there exists $i_{0} \in\{1, \ldots, m\}$ such that $x-a_{i_{0}} \notin \operatorname{cone}\left(C_{a_{i_{0}}}\right)$ and thus $\varphi_{a_{i_{0}}}\left(x-a_{i_{0}}\right)=+\infty$. Therefore, since the unit ball of $\gamma$ is bounded, $F(x)=+\infty \quad \forall x \in \mathbb{R}^{n}$.

Conversely, if $F^{*}=+\infty$, then $F(x)=+\infty \quad \forall x \in \mathbb{R}^{n}$. Thus, since the unit ball of $\gamma$ contains the origin in its interior, for all $x \in \mathbb{R}^{n}$ there exists 
$i_{0}(x) \in\{1, \ldots, m\} \quad$ such that $\quad \varphi_{a_{i_{0}(x)}}\left(x-a_{i_{0}(x)}\right)=+\infty \quad$ and then $x \in \mathbb{R}^{n} \backslash$ cone $\left(C_{a_{i_{0}(x)}}\right)$. Therefore, the condition holds.

Notice that condition (13) only holds if the origin is in the boundary of at least two unit balls of the gauges associated with the existing facilities. Otherwise, there are at least $m-1$ facilities (assume without loss of generality indexed by $i=1, \ldots, m-1)$ such that cone $\left(C_{a_{i}}\right)=\mathbb{R}^{n}$ for all $i=1, \ldots, m-1$. Then, $\bigcap_{i=1}^{m}\left(a_{i}+\operatorname{cone}\left(C_{a_{i}}\right)\right)$ can not be the empty set.

Lemma 5. Suppose that the globalizing function $\gamma$ is the gauge of an unbounded closed convex set. Then, the optimal objective value for Problem $P_{w}^{\gamma}(A)$ is unbounded if the following condition holds:

$$
\forall x \in \mathbb{R}^{n} \quad\left(w_{a_{1}} \varphi_{a_{1}}\left(x-a_{1}\right), \ldots, w_{a_{m}} \varphi_{a_{m}}\left(x-a_{m}\right)\right) \in \mathbb{R}^{m} \backslash \operatorname{cone}\left(C_{\gamma}\right) .
$$

Proof. We know that if $x \notin \operatorname{cone}\left(C_{\gamma}\right)$, then $\gamma(x)=+\infty$ and therefore the result follows.

Notice that condition (14) is a sufficient but not necessary condition for $F^{*}=+\infty$. We can see this assertion in the following example.

Example 3. We are given two existing facilities $a_{1}=(-1,0)$ and $a_{2}=(1,0)$ in $\mathbb{R}^{2}$. The unit ball of $\varphi_{a_{1}}$ is the halfspace $C_{a_{1}}=\left\{(x, y) \in \mathbb{R}^{2}: x \leq 0\right\}$ and the unit ball of $\varphi_{a_{2}}$ is the halfspace $C_{a_{2}}=\left\{(x, y) \in \mathbb{R}^{2}: x \geq 0\right\}$. Let $\gamma$ be the polyhedral gauge in $\mathbb{R}^{2}$ whose unit ball is $C_{\gamma}=\left\{(x, y) \in \mathbb{R}^{2}: x \leq 1 ; y \leq 1\right\}$ (see Figure 3). Notice that cone $\left(C_{\gamma}\right)=\mathbb{R}^{2}$. Nevertheless,

- if $x \leq-1$, then $\varphi_{a_{1}}(x, y)=0$ and $\varphi_{a_{2}}(x, y)=+\infty \quad \forall y \in \mathbb{R}$;

- if $-1<x<1$, then $\varphi_{a_{1}}(x, y)=+\infty$ and $\varphi_{a_{2}}(x, y)=+\infty \quad \forall y \in \mathbb{R}$;

- if $x \geq 1$, then $\varphi_{a_{1}}(x, y)=+\infty$ and $\varphi_{a_{2}}(x, y)=0 \quad \forall y \in \mathbb{R}$.

Therefore, $F(x, y)=+\infty \quad \forall(x, y) \in \operatorname{cone}\left(C_{\gamma}\right)$.

In the next section we study the non-degenerate cases. Then, we assume that conditions (11), (12), (13) and (14) are not fulfilled.

\section{Geometrical description of optimal sets}

In this section we look for a geometrical characterization of the set of optimal solutions $M_{w}^{\gamma}(A)$ of the problem $P_{w}^{\gamma}(A)$ introduced in Section 3. In doing that,
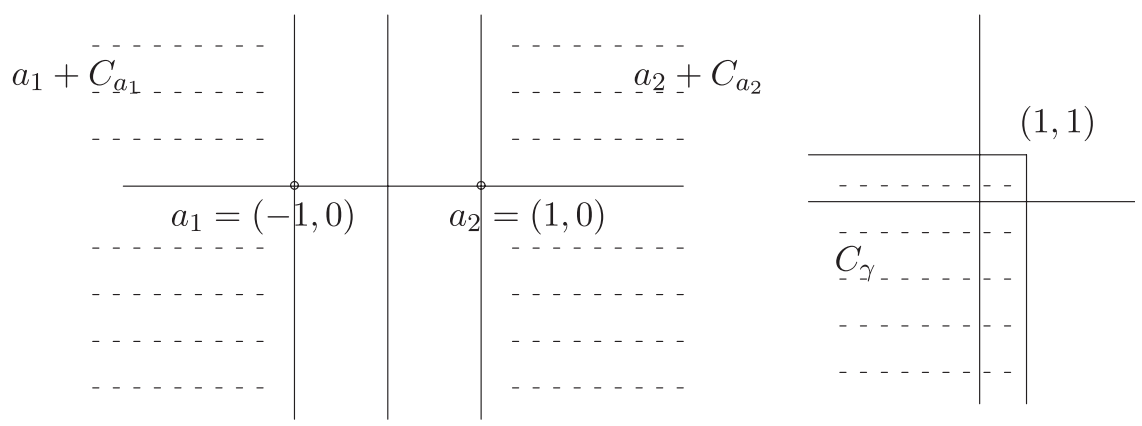

Fig. 3. Counter-example 
we recall the concept of an elementary convex set (see Durier and Michelot [4]). Through this section we will assume that $F^{*}$ is different from 0 and $+\infty$, otherwise it will be stated explicitly.

Definition 2. Let $\pi=\left(p_{a}\right)_{a \in A}$ be a family of elements of $\mathbb{R}^{n}$ such that $p_{a} \in C_{a}^{*}$ for each $a \in A$; let $N_{a}\left(p_{a}\right)$ be the normal cone to $C_{a}^{*}$ at $p_{a}$; and let

$$
\mathcal{C}_{\pi}=\bigcap_{a \in A}\left(a+N_{a}\left(p_{a}\right)\right) .
$$

A nonempty convex set $\mathcal{C}$ is said to be an elementary convex set if there exists a family $\pi$ such that $\mathcal{C}_{\pi}=\mathcal{C}$.

As an illustration of this definition we show some examples in Figure 4. Notice that if $\varphi$ is a quadratic gauge of an unbounded closed convex set and $\varphi_{a}(x)=\varphi(x)$ for all $a \in A$, then the elementary convex sets are parallel strips or lines passing through the existing facilities. If $\varphi_{a}(x) \neq \varphi_{b}(x)$ with $a \neq b$, the elementary convex sets are polyhedra, their facets and their vertices. Obtaining the elementary convex sets for the third case, where the polyhedral gauge was described in Figure 2, only consists of computing the normal cone to $C^{*}$ at any point of its boundary. In this case the elementary convex sets are polyhedra, their facets and some of their vertices. It is worth noting that the existing facilities may not be elementary convex sets.

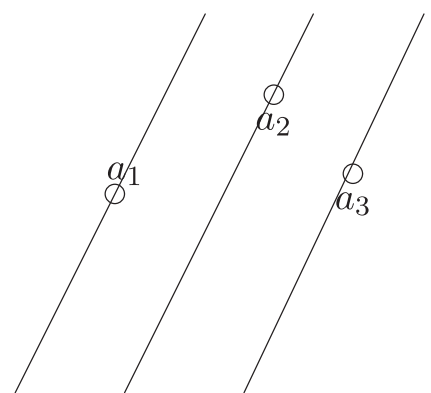

The same quadratic gauge

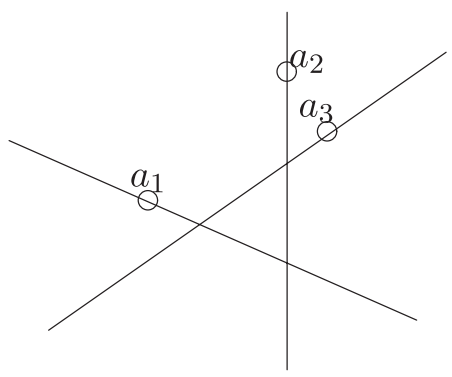

Different quadratic gauges

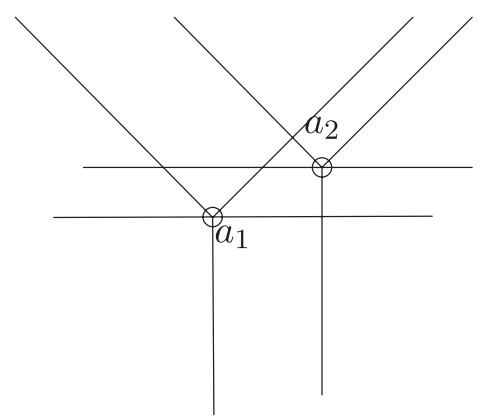

The polyhedral gauge of Figure 2

Fig. 4. Examples of elementary convex sets 
In order to give a geometrical description of the set $M_{w}^{\gamma}(A)$ we will use the condition: $x \in M_{w}^{\gamma}(A)$ if and only if $0 \in \partial F(x)$. Thus, we start with a characterization of the subdifferential set $\partial F(x)$, which makes use of the Theorem VI.4.3.1 in Hiriart-Urruty and Lemaréchal [10]. This result requires monotonicity of the outer component of the composition between the functions. We recall that a function $f$ on $\mathbb{R}^{m}$ is said to be monotone if $f(u) \leq f(v)$ for every $u, v \in \mathbb{R}^{m}$ verifying $u_{i} \leq v_{i} \quad \forall i=1, \ldots, m$. Notice that a monotone gauge on $\mathbb{R}^{m}$ is not a monotone function in the above sense. For this reason we will introduce the function $\gamma^{+}$.

Let $Q$ and $\bar{Q}$ be two sets in $\mathbb{R}^{m}$ defined by $Q=\left\{u \in \mathbb{R}^{m}: u_{i}>0\right.$ $\forall i=1, \ldots, m\}$ and $\bar{Q}=\left\{u \in \mathbb{R}^{m}: u_{i} \geq 0 \quad \forall i=1, \ldots, m\right\}$. We assume $\gamma$ to be a monotone gauge on $Q$, i.e., $\gamma(u) \leq \gamma(v)$ for every $u$ and $v$ in $\mathbb{R}^{m}$ satisfying $0 \leq u_{i} \leq v_{i}$ for each $i=1, \ldots, m$.

Proposition 1. Let $\gamma$ be a monotone gauge on $\bar{Q}$. Then, the function

$$
\gamma^{+}(u)=\gamma\left(\max \left(u_{1}, 0\right), \ldots, \max \left(u_{m}, 0\right)\right)
$$

is a sublinear, monotone function in $\mathbb{R}^{m}$. Moreover, $\partial \gamma^{+}(u) \subset \bar{Q} \quad \forall u \in \bar{Q}$.

The proof runs parallel to Proposition 3.6 in Durier [5] and therefore is omitted.

Since $\gamma^{+}$is a sublinear function, $\gamma^{+}$is the support function of the polar set of its "unit ball". Let $B=\left\{u \in \mathbb{R}^{m}: \gamma^{+}(u) \leq 1\right\}$ be the unit ball of $\gamma^{+}$, and let $B^{*}$ be the polar set of $B$. The subdifferential set of the function $\gamma^{+}$at a point $u \in \bar{Q}, \quad u \neq 0$ is

$$
\begin{aligned}
\partial \gamma^{+}(u)=\left\{\lambda=\left(\lambda_{1}, \ldots, \lambda_{m}\right): \lambda_{i} \geq 0 \quad\right. & \forall i=1, \ldots, m, \lambda \in B^{*}, \\
& \left.\langle\lambda, u\rangle=\gamma^{+}(u)\right\} .
\end{aligned}
$$

Lemma 6. An element $x^{*} \in \mathbb{R}^{n}$ belongs to $\partial F(x)$ if and only if there exist $p_{a_{1}} \in$ $C_{a_{1}}^{*}, \ldots, p_{a_{m}} \in C_{a_{m}}^{*}$ and $\lambda=\left(\lambda_{1}, \ldots, \lambda_{m}\right) \in B^{*}$ with $\lambda_{i} \geq 0 \forall i=1, \ldots, m$ such that $\quad x \in a_{i}+N_{a_{i}}\left(p_{a_{i}}\right) \forall i=1, \ldots, m, \quad \sum_{i=1}^{m} \lambda_{i} w_{a_{i}} \varphi_{a_{i}}\left(x-a_{i}\right)=F(x), \quad$ and $x^{*}=\sum_{i=1}^{m} \lambda_{i} w_{a_{i}} p_{a_{i}}$.

Proof. Let $\Phi(x)=\left(w_{a_{1}} \varphi_{a_{1}}\left(x-a_{1}\right), \ldots, w_{a_{m}} \varphi_{a_{m}}\left(x-a_{m}\right)\right)$, then $F(x)=\left(\gamma^{+} \circ \Phi\right)(x), F$ being a convex function. Thus, by Theorem VI.4.3.1 in Hiriart-Urruty and Lemaréchal [10] we have that

$$
\begin{aligned}
\partial F(x)=\left\{\sum_{i=1}^{m} \lambda_{i} q_{a_{i}}:\left(\lambda_{1}, \ldots, \lambda_{m}\right) \in \partial \gamma^{+}(\Phi(x)),\right. \\
\left.q_{a_{i}} \in \partial\left(w_{a_{i}} \varphi_{a_{i}}\left(x-a_{i}\right)\right) \forall i=1, \ldots, m\right\} .
\end{aligned}
$$

On the other hand, we know from (17) that $\partial \gamma^{+}(\Phi(x))=\{\lambda=$ $\left.\left(\lambda_{1}, \ldots, \lambda_{m}\right): \lambda \in B^{*}, \lambda_{i} \geq 0 \forall i=1, \ldots, m,\langle\lambda, \Phi(x)\rangle=\gamma^{+}(\Phi(x))=F(x)\right\}$.

Finally, $q_{a_{i}}=w_{a_{i}} p_{a_{i}}$ with $p_{a_{i}} \in \partial \varphi_{a_{i}}\left(x-a_{i}\right)$ and $\partial \varphi_{a_{i}}\left(x-a_{i}\right)=\left\{p_{a_{i}} \in C_{a_{i}}^{*}\right.$ : $\left.x \in a_{i}+N_{a_{i}}\left(p_{a_{i}}\right)\right\}$ for all $i=1, \ldots, m$. Thus, the result follows.

Let us now introduce some notation. For $J \subseteq\{1, \ldots, m\}, J \neq \emptyset$, and $p=\left(p_{a_{i}}\right)_{i \in J}$ with $p_{a_{i}} \in C_{a_{i}}^{*}$ let 


$$
\begin{aligned}
C_{J}(p) & =\left\{x \in \mathbb{R}^{n}:\left\langle p_{a_{i}}, x-a_{i}\right\rangle=\varphi_{a_{i}}\left(x-a_{i}\right) \forall i \in J\right\} \\
& =\bigcap_{i \in J}\left(a_{i}+N_{a_{i}}\left(p_{a_{i}}\right)\right),
\end{aligned}
$$

and for $\lambda=\left(\lambda_{1}, \ldots, \lambda_{m}\right) \in B^{*}$ let

$$
\begin{aligned}
D_{J}(\lambda) & =\left\{x \in \mathbb{R}^{n}: F(x)=-\sum_{i \in J} \lambda_{i} w_{a_{i}}\left\langle p_{a_{i}}, a_{i}\right\rangle\right\} \\
& =\left\{x \in \mathbb{R}^{n}: F(x)=\sum_{i \in J} \lambda_{i} w_{a_{i}} \varphi_{a_{i}}\left(x-a_{i}\right)\right\} .
\end{aligned}
$$

Lemma 7. A point $x \in \mathbb{R}^{n}$ belongs to $M_{w}^{\gamma}(A)$ if and only if there exist $J \subseteq\{1, \ldots, m\}, J \neq \emptyset, p=\left(p_{a_{i}}\right)_{i \in J}$ with $p_{a_{i}} \in C_{a_{i}}^{*}$, and $\lambda=\left(\lambda_{1}, \ldots, \lambda_{m}\right) \in B^{*}$ with $\lambda_{i}>0 \quad \forall i \in J$ and $\lambda_{i}=0 \quad \forall i \notin J$ satisfying $\sum_{i \in J} \lambda_{i} w_{a_{i}} p_{a_{i}}=0$ such that $x \in C_{J}(p) \cap D_{J}(\lambda)$.

Proof. Let $x \in \mathbb{R}^{n}$. We know that $x \in M_{w}^{\gamma}(A)$ if and only if $0 \in \partial F(x)$. Therefore, by Lemma 6 there exist $p_{a_{1}} \in C_{a_{1}}^{*}, \ldots, p_{a_{m}} \in C_{a_{m}}^{*}$, $\lambda=\left(\lambda_{1}, \ldots, \lambda_{m}\right) \in B^{*}$ with $\lambda_{i} \geq 0 \quad \forall i=1, \ldots, m$ such that

$$
\begin{aligned}
& x \in a_{i}+N_{a_{i}}\left(p_{a_{i}}\right) \quad \forall i=1, \ldots, m, \\
& \sum_{i=1}^{m} \lambda_{i} w_{a_{i}} \varphi_{a_{i}}\left(x-a_{i}\right)=F(x), \text { and } \\
& 0=\sum_{i=1}^{m} \lambda_{i} w_{a_{i}} p_{a_{i}} .
\end{aligned}
$$

The assertion (23) implies that $\left\langle p_{a_{i}}, x-a_{i}\right\rangle=\varphi_{a_{i}}\left(x-a_{i}\right) \forall i=1, \ldots, m$. Then, by (24) we have that $F(x)=\sum_{i=1}^{m} \lambda_{i} w_{a_{i}}\left\langle p_{a_{i}}, x\right\rangle-\sum_{i=1}^{m} \lambda_{i} w_{a_{i}}\left\langle p_{a_{i}}, a_{i}\right\rangle$ and by (25) we obtain $F(x)=-\sum_{i \in J} \lambda_{i} w_{a_{i}}\left\langle p_{a_{i}}, a_{i}\right\rangle$. Let $J=\left\{i: \lambda_{i}>0\right\}$, then $x \in C_{J}(p) \cap D_{J}(\lambda)$.

Note that $J \neq \emptyset$ because if $\lambda_{i}=0 \forall i=1, \ldots, m$, then $F(x)=0$ and we have assumed that $F^{*} \neq 0$.

The next theorem deals with the simultaneous characterization of all the points of the optimal solution set $M_{w}^{\gamma}(A)$. Once we have proved the previous technical lemmas the proof runs parallel to Theorem 4.3 in Durier [2] and therefore is omitted.

\section{Theorem 2}

1. If $M_{w}^{\gamma}(A)$ is nonempty, then there exist $J \subseteq\{1, \ldots, m\}, J \neq \emptyset,\left(p_{a_{i}}\right)_{i \in J}$ with $p_{a_{i}} \in C_{a_{i}}^{*}$ and $\lambda=\left(\lambda_{1}, \ldots, \lambda_{m}\right) \in B^{*}$ with $\lambda_{i}>0 \quad \forall i \in J$ and $\lambda_{i}=0 \quad \forall i \notin J$ satisfying $\sum_{i \in J} \lambda_{i} w_{a_{i}} p_{a_{i}}=0$ such that

$$
M_{w}^{\gamma}(A)=C_{J}(p) \cap D_{J}(\lambda) .
$$

2. If there exist $J \subseteq\{1, \ldots, m\}, J \neq \emptyset,\left(p_{a_{i}}\right)_{i \in J}$ with $p_{a_{i}} \in C_{a_{i}}^{*}$ and $\lambda=\left(\lambda_{1}, \ldots, \lambda_{m}\right) \in B^{*}$ with $\lambda_{i}>0 \quad \forall i \in J$ and $\lambda_{i}=0 \quad \forall i \notin J$ satisfying $\sum_{i \in J} \lambda_{i} w_{a_{i}} p_{a_{i}}=0$ such that $C_{J}(p) \cap D_{J}(\lambda) \neq \emptyset$, then

$$
M_{w}^{\gamma}(A)=C_{J}(p) \cap D_{J}(\lambda) .
$$


This theorem shows that the set of optimal solutions of our problem can be obtained as the intersection between two sets: the first one is an elementary convex set and the second one is the set where the objective function admits a linear representation as function of its arguments.

Example 4. We are given three existing facilities $A=\left\{a_{1}, a_{2}, a_{3}\right\}$ on the plane. Assume that $\varphi_{a_{i}}(x) i=1,2,3$ are quadratic gauges and that there exist $i \neq j$ with $\varphi_{a_{i}}(x) \neq \varphi_{a_{j}}(x)$. We want to find the points minimizing the sum of the weighted "distances" to the three given facilities. Thus, the problem is formulated as

$$
\min \sum_{i=1}^{3} w_{a_{i}} \varphi_{a_{i}}\left(x-a_{i}\right)
$$

Notice that, since the asymptotic cone $\left(C_{a_{i}}\right)_{\infty}$ of $\varphi_{a_{i}}$ is a straight line passing through $a_{i}$ for all $i=1,2,3$, this problem is equivalent to find the points that minimize the sum of the weighted Euclidean distances to three given straight lines.

In order to solve this problem, notice that the globalizing function $\gamma$ is the $l_{1}$-norm. Then, $B^{*}$ is the unit ball of the $l_{\infty}$-norm. Thus, there exist $\lambda=(1,1,1) \in B^{*}, \quad J=\{1,2,3\}, \quad$ and $\quad p_{a_{i}} \in C_{a_{i}}^{*}$ for $i=1,2,3$ satisfying $\sum_{i=1}^{3} w_{a_{i}} p_{a_{i}}=0$ such that

$$
M_{w}^{l_{1}}(A)=C_{J}(p) \cap D_{J}(\lambda)=C_{J}(p) \cap \mathbb{R}^{2}=C_{J}(p)=\bigcap_{i=1}^{3}\left(a_{i}+N_{a_{i}}\left(p_{a_{i}}\right)\right) .
$$

Therefore, the optimal solution set is an elementary convex set. In addition, the function $\varphi_{a_{i}}\left(x-a_{i}\right)$ increases in the direction orthogonal to the line $a_{i}+\left(C_{a_{i}}\right)_{\infty}$ for all $i=1,2,3$. Thus, since $\varphi_{a_{i}}(x) \neq \varphi_{a_{i}}(x)$ for some $i \neq j$, $M_{w}^{l_{1}}(A)$ has to be a bounded elementary convex set. Otherwise, $\varphi_{a_{i_{0}}}\left(x-a_{i_{0}}\right)$ goes to infinity for some $i_{0}=1,2,3$.

- Suppose $\varphi_{a_{i}} \neq \varphi_{a_{j}} \quad \forall i \neq j$ with $i, j=1,2,3$. Two subcases can occur:

- The lines $a_{i}+\left(C_{a_{i}}\right)_{\infty}$ for $i=1,2,3$ intersect at a single point. Then, by Lemma 2 this point is the minimizer and the optimal objective value is zero.

- The lines $a_{i}+\left(C_{a_{i}}\right)_{\infty}$ for $i=1,2,3$ intersect delimiting a triangle. In this case, the bounded elementary convex sets are: 1) that triangle; 2) its facets; and 3) its vertices.

The optimal point is that vertex giving the smaller objective function value. If two vertices give the same minimum value the whole facet is optimal. If the three vertices attain the same objective value the optimal solution set is the triangle itself.

- Suppose $\varphi_{a_{1}}=\varphi_{a_{2}} \neq \varphi_{a_{3}}$. In this case, the bounded elementary convex sets are: 1) the segment on the line $a_{3}+\left(C_{a_{3}}\right)_{\infty}$ bounded by the lines $a_{1}+\left(C_{a_{1}}\right)_{\infty}$ and $a_{2}+\left(C_{a_{2}}\right)_{\infty}$; and 2) its extreme points.

If the objective function value is the same at both extreme points the optimal solution set is the whole segment. Otherwise, the optimal point is that extreme point giving the smaller objective function value. 
Two remarkable applications (consequences) of Theorem 2 lead to two characterizations of optimal solution sets of well-known problems in Location Theory: The Weber problem and the minimax problem.

\subsection{The Weber problem with gauges of closed convex sets}

Let $\varphi_{a_{i}}$ be the gauge of a closed (not necessarily bounded) convex set $C_{a_{i}}$ containing the origin. The mathematical formulation of the Weber problem is

$$
P_{w}^{l_{1}}(A) \quad \min _{x \in \mathbb{R}^{n}} F(x)=\sum_{i=1}^{m} w_{a_{i}} \varphi_{a_{i}}\left(x-a_{i}\right) .
$$

This problem was already solved by Durier and Michelot [4] for functions $\varphi_{a_{i}}$ being gauges of compact convex sets. The results obtained by Durier and Michelot are still valid for gauges of closed convex sets except for the fact that the optimal solution set $M_{w}^{l_{1}}(A)$ need not be a bounded set. In addition, as we have already seen before, the optimal objective value may be zero or unbounded.

Corollary 1. If $\bigcap_{i=1}^{m}\left(a_{i}+\operatorname{cone}\left(C_{a_{i}}\right)\right) \neq \emptyset$, then $M_{w}^{l_{1}}(A)$ is a closed elementary convex set $\mathcal{C}_{\pi}$ given by a family $\pi=\left(p_{a_{i}}\right)_{a_{i} \in A}$ such that $\sum_{i=1}^{m} w_{a_{i}} p_{a_{i}}=0$. Conversely, let $\mathcal{C}_{\pi}$ be an elementary convex set associated with a family $\pi=\left(p_{a_{i}}\right)_{a_{i} \in A}$ such that $\sum_{i=1}^{m} w_{a_{i}} p_{a_{i}}=0$, then $\mathcal{C}_{\pi}=M_{w}^{l_{1}}(A)$.

Proof. Notice that the globalizing function $\gamma$ is the $l_{1}$-norm. Then, if $\bigcap_{i=1}^{m}\left(a_{i}+\operatorname{cone}\left(C_{a_{i}}\right)\right) \neq \emptyset$, by Lemma $4 M_{w}^{l_{1}}(A) \neq \emptyset$. On the other hand, $B^{*}$ is the unit ball of the $l_{\infty}$-norm. Thus, taking $\lambda=(1, \ldots, 1) \in B^{*}$ and $J=\{1, \ldots, m\}$ we have that $D_{J}(\lambda)=\mathbb{R}^{n}$. Therefore, by Theorem 2 the optimal solution set reduces to a closed elementary convex set given by a family $\left(p_{a_{i}}\right)_{i=1, \ldots, m}$ such that $\sum_{i=1}^{m} w_{a_{i}} p_{a_{i}}=0$.

Notice that $M_{w}^{l_{1}}(A)$ need not be compact. For instance, if $\bigcap_{i=1}^{m}\left(a_{i}+\left(C_{a_{i}}\right)_{\infty}\right) \neq \emptyset$, it may exist $\left\{x_{n}\right\}_{n \in \mathbb{N}} \subset \bigcap_{i=1}^{m}\left(a_{i}+\left(C_{a_{i}}\right)_{\infty}\right)$ with $\left\|x_{n}\right\| \longrightarrow+\infty$ if $n \longrightarrow+\infty$ and in addition, by Lemma 2 the optimal objective value is zero.

In the special case that all the gauges are polyhedral we can solve the Weber problem using a natural reformulation as a linear program.

\section{The Weber problem with polyhedral gauges}

Assume that $\varphi_{a_{i}}$ is the gauge of a closed convex polyhedron $C_{a_{i}}$ for all $i=1, \ldots, m$. Thus, the polar set $C_{a_{i}}^{*}$ is a closed convex polyhedron as well (see Corollary 19.2.2 in Rockafellar [17]), and then $C_{a_{i}}^{*}$ has a finite number of extreme points and extreme directions (see Corollary 19.1.1 in Rockafellar [17]).

As we have seen in (5), $\varphi_{a_{i}}$ is the support function of $C_{a_{i}}^{*}$. First consider the case where $C_{a_{i}}^{*}$ has zero linearity. Therefore, $\varphi_{a_{i}}$ can be written as (see Example V.3.4.3 in Hiriart-Urruty and Lemaréchal [10])

$$
\varphi_{a_{i}}(x)= \begin{cases}\max _{b_{a_{i}} \in \operatorname{ext}\left(C_{a_{i}}^{*}\right)}\left\langle b_{a_{i}}, x\right\rangle & \text { if }\left\langle d_{a_{i}}, x\right\rangle \leq 0 \quad \forall d_{a_{i}} \in\left(C_{a_{i}}^{*}\right)_{\infty}^{\text {ext }}, \\ +\infty & \text { otherwise }\end{cases}
$$


where $\operatorname{ext}\left(C_{a_{i}}^{*}\right)$ and $\left(C_{a_{i}}^{*}\right)_{\infty}^{\text {ext }}$ stand for the set of extreme points and extreme directions of $C_{a_{i}}^{*}$, respectively.

Now suppose that $C_{a_{i}}^{*}$ has non-zero linearity. By Lemma $1 C_{a_{i}}^{*}=C_{a_{i} 0}^{*}+L_{a_{i}}^{*}$ where $L_{a_{i}}^{*}$ is the linearity subspace of $C_{a_{i}}^{*}$ and $C_{a_{i} 0}^{*}=C_{a_{i}}^{*} \cap L_{a_{i}}^{* \perp}$. It is straightforward to see that if $C_{a_{i}}^{*}$ is a polyhedron, $C_{a_{i} 0}^{*}$ is also a polyhedron, and then $C_{a_{j} 0}^{*}$ has a finite number of extreme points and extreme directions. On the other hand, a necessary condition for $\varphi_{a_{i}}(x)<+\infty$ is that $x \in L_{a_{i}}^{* \perp}$ (see Theorem 14.6 in Rockafellar [17]). Thus, $\varphi_{a_{i}}$ can be written as

$$
\varphi_{a_{i}}(x)= \begin{cases}\max _{b_{a_{i}} \in \operatorname{ext}\left(C_{a_{i} 0}^{*}\right)}\left\langle b_{a_{i}}, x\right\rangle & \text { if }\left\langle d_{a_{i}}, x\right\rangle \leq 0 \quad \forall d_{a_{i}} \in\left(C_{a_{i} 0}^{*}\right)_{\infty}^{\text {ext }}, \\ +\infty & \text { and }\left\langle v_{a_{i}}, x\right\rangle=0 \quad \forall v_{a_{i}} \in B_{L_{a_{i}}^{*}}, \\ \text { otherwise }\end{cases}
$$

where $B_{L_{a_{i}}^{*}}$ is a basis of $L_{a_{i}}^{*}$, and $\operatorname{ext}\left(C_{a_{i} 0}^{*}\right)$ and $\left(C_{a_{i} 0}^{*}\right)_{\infty}^{e x t}$ stand for the set of extreme points and extreme directions of $C_{a_{i} 0}^{*}$, respectively.

Let $z_{a_{i}}$ be an auxiliary variable such that

$$
\max \left\{\left\langle b_{a_{i}}, x-a_{i}\right\rangle: b_{a_{i}} \in \operatorname{ext}\left(C_{a_{i} 0}^{*}\right)\right\} \leq z_{a_{i}} .
$$

Then, Problem $P_{w}^{l_{1}}(A)$ with polyhedral gauges can be written as

$$
\begin{aligned}
\min & \sum_{i=1}^{m} w_{a_{i}} z_{a_{i}} \\
\text { s.t. }\left\langle b_{a_{i}}, x-a_{i}\right\rangle \leq z_{a_{i}} & \forall b_{a_{i}} \in \operatorname{ext}\left(C_{a_{i} 0}^{*}\right), \text { and } \forall i=1, \ldots, m, \\
\left\langle d_{a_{i}}, x-a_{i}\right\rangle \leq 0 & \forall d_{a_{i}} \in\left(C_{a_{i} 0}^{*}\right)_{\infty}^{e x t}, \text { and } \forall i=1, \ldots, m, \\
\left\langle v_{a_{i}}, x-a_{i}\right\rangle=0 & \forall v_{a_{i}} \in B_{L_{a_{i}}^{*}}, \text { and } \forall i=1, \ldots, m .
\end{aligned}
$$

This problem can be solved with any linear programing algorithm. In particular, interior point algorithms provide polynomial time resolution methods. Notice that similar LP-formulations have been obtained for other location problems with polyhedral gauges of compact convex sets. The interested reader is referred to Rodriguez-Chia et al. [18] and the references therein.

On the other hand, we can also obtain another reformulation of Problem $P_{w}^{l_{1}}(A)$ with polyhedral gauges as a linear program making use of the geometrical interpretation of any gauge given by Theorem 1 .

Let $C_{a_{i}}$ be a closed convex polyhedron, with linearity subspace $L_{a_{i}}$, and let $C_{a_{i} 0}=C_{a_{i}} \cap L_{a_{i}}^{\perp}$. Suppose the extreme points of $C_{a_{i} 0}$ are $\operatorname{ext}\left(C_{a_{i} 0}\right):=$ $\left\{b_{a_{i}}^{1}, \ldots, b_{a_{i}}^{R_{i}}\right\}$, the extreme directions of $C_{a_{i} 0}$ are $\left(C_{a_{i} 0}\right)_{\infty}^{\text {ext }}:=\left\{d_{a_{i}}^{1}, \ldots, d_{a_{i}}^{J_{i}}\right\}$, and $\left\{v_{a_{i}}^{1}, \ldots, v_{a_{i}}^{p_{i}}\right\}$ is a basis of $L_{a_{i}}$. By Theorem 1 we know that

$$
\begin{aligned}
\varphi_{a_{i}}\left(x-a_{i}\right)= & \min \sum_{r=1}^{R_{i}} \lambda_{a_{i}}^{r} \\
\text { s.t. } x-a_{i}= & \sum_{r=1}^{R_{i}} \lambda_{a_{i}}^{r} b_{a_{i}}^{r}+\sum_{j=1}^{J_{i}} \mu_{a_{i}}^{j} d_{a_{i}}^{j}+\sum_{k=1}^{p_{i}} \delta_{a_{i}}^{k} v_{a_{i}}^{k}, \\
& \lambda_{a_{i}}^{r}, \mu_{a_{i}}^{j} \geq 0 \forall i, r, j, \text { and } \delta_{a_{i}}^{k} \in \mathbb{R}, \forall k .
\end{aligned}
$$


Therefore, we can rewrite Problem $P_{w}^{l_{1}}(A)$ with polyhedral gauges as

$$
\begin{aligned}
& \min \sum_{i=1}^{m} \sum_{r=1}^{R_{i}} w_{a_{i}} \lambda_{a_{i}}^{r} \\
& \text { s.t. } x-a_{i}=\sum_{r=1}^{R_{i}} \lambda_{a_{i}}^{r} b_{a_{i}}^{r}+\sum_{j=1}^{J_{i}} \mu_{a_{i}}^{j} d_{a_{i}}^{j}+\sum_{k=1}^{p_{i}} \delta_{a_{i}}^{k} v_{a_{i}}^{k} \quad \forall i=1, \ldots, m, \\
& \quad \lambda_{a_{i}}^{r}, \mu_{a_{i}}^{j} \geq 0 \forall i, r, j, \text { and } \delta_{a_{i}}^{k} \in \mathbb{R} \forall k .
\end{aligned}
$$

\subsection{The minimax problem with gauges of closed convex sets}

A second consequence of Theorem 2 is the geometrical description of the set of optimal solutions for the particular case of the minimax problem. The mathematical formulation of the problem is

$$
P_{w}^{l_{\infty}}(A) \quad \min _{x \in \mathbb{R}^{n}} F(x)=\max _{i=1, \ldots, m} w_{a_{i}} \varphi_{a_{i}}\left(x-a_{i}\right) .
$$

We introduce the following notation. For $J \subseteq\{1, \ldots, m\}, \quad J \neq \emptyset$, and $\tau>0$ we let

$$
\begin{aligned}
E_{J}(\tau)= & \left\{x \in \mathbb{R}^{n}: w_{a_{i}} \varphi_{a_{i}}\left(x-a_{i}\right)=\tau \forall i \in J,\right. \text { and } \\
& \left.w_{a_{i}} \varphi_{a_{i}}\left(x-a_{i}\right) \leq \tau \forall i \notin J\right\} .
\end{aligned}
$$

\section{Corollary 2}

1. If $\bigcap_{i=1}^{m}\left(a_{i}+\operatorname{cone}\left(C_{a_{i}}\right)\right) \neq \emptyset$, then there exist $\tau>0$, $J \subseteq\{1, \ldots, m\}, J \neq \emptyset,\left(p_{a_{i}}\right)_{i \in J}$ with $p_{a_{i}} \in C_{a_{i}}^{*}$, and $\lambda=\left(\lambda_{1}, \ldots, \lambda_{m}\right)$ with $\lambda_{i}>0 \forall i \in J \quad$ and $\quad \lambda_{i}=0 \forall i \notin J \quad$ satisfying $\quad \sum_{i \in J} \lambda_{i}=1$, and $\sum_{i=1}^{m} \lambda_{i} w_{a_{i}} p_{a_{i}}=0$ such that $M_{w}^{l_{\infty}}(A)=C_{J}(p) \cap E_{J}(\tau)$. Moreover, $\tau$ is the optimal value of $F$ and $\tau=-\sum_{i \in J} \lambda_{i} w_{a_{i}}\left\langle p_{a_{i}}, a_{i}\right\rangle$.

2. If there exist $\tau>0, J \subseteq\{1, \ldots, m\}, J \neq \emptyset,\left(p_{a_{i}}\right)_{i \in J}$ with $p_{a_{i}} \in C_{a_{i}}^{*}$, and $\lambda=$ $\left(\lambda_{1}, \ldots, \lambda_{m}\right) \in B^{*} \quad$ with $\lambda_{i}>0 \forall i \in J$, and $\lambda_{i}=0 \forall i \notin J$ satisfying $\sum_{j \in J} \lambda_{i}=1$, and $\sum_{i=1}^{m} \lambda_{i} w_{a_{i}} p_{a_{i}}=0$ such that $C_{J}(p) \cap E_{J}(\tau) \neq \emptyset$, then $M_{w}^{t_{\infty}}(A)=C_{J}(p) \cap E_{J}(\tau)$, and $\tau=-\sum_{i \in J} \lambda_{i} w_{a_{i}}\left\langle p_{a_{i}}, a_{i}\right\rangle$ is the optimal value of $F$.

Proof. Notice that the globalizing function $\gamma$ is the $l_{\infty}$-norm. Then, if $\bigcap_{i=1}^{m}\left(a_{i}+\operatorname{cone}\left(C_{a_{i}}\right)\right) \neq \emptyset$, by Lemma $4 M_{w}^{l_{1}}(A) \neq \emptyset$. On the other hand, $B^{*}$ is the unit ball of the $l_{1}$-norm. Therefore, $\lambda_{i}>0 \forall i \in J, \lambda_{i}=0 \forall i \notin J$, and $\sum_{i \in J} \lambda_{i}=1$ implies $\lambda \in B^{*}$. Thus, if $\sum_{i \in J} \lambda_{i}=1$, the condition $w_{a_{i}} \varphi_{a_{i}}\left(x-a_{i}\right)=\tau \forall i \in J$ and $w_{a_{i}} \varphi_{a_{i}}\left(x-a_{i}\right) \leq \tau \forall i \notin J$ is equivalent to $F(x)=\tau=\sum_{i \in J} \lambda_{i} \tau=\sum_{i \in J} \lambda_{i} w_{a_{i}} \varphi_{a_{i}}\left(x-a_{i}\right)$. Hence, the proof can be obtained by Theorem 2 taking into account that in this case $D_{J}(\lambda)=E_{J}(\tau)$ for $\tau=F(x)$.

In the special case that all the gauges are polyhedral we can also solve the minimax problem using a natural reformulation as a linear program. 


\section{The minimax problem with polyhedral gauges}

Assume the same notation as in (27). It is easy to see that the resolution of Problem $P_{w}^{l_{\infty}}(A)$ with polyhedral gauges reduces to solve the following equivalent problem:

$\min z$

$$
\begin{array}{ll}
\text { s.t. } & w_{a_{i}}\left\langle b_{a_{i}}, x-a_{i}\right\rangle \leq z \quad \forall b_{a_{i}} \in \operatorname{ext}\left(C_{a_{i} 0}^{*}\right), \text { and } \forall i=1, \ldots, m, \\
& \left\langle d_{a_{i}}, x-a_{i}\right\rangle \leq 0 \quad \forall d_{a_{i}} \in\left(C_{a_{i} 0}^{*}\right)_{\infty}^{e x t}, \text { and } \forall i=1, \ldots, m, \\
& \left\langle v_{a_{i}}, x-a_{i}\right\rangle=0 \quad \forall v_{a_{i}} \in B_{L_{a_{i}}^{*}}, \text { and } \forall i=1, \ldots, m .
\end{array}
$$

\section{Concluding remarks}

In this paper we deal with a new approach of single facility location problems based on gauges of unbounded closed convex sets. This analysis generalizes previous approaches based on gauges of bounded sets. Geometrically this problems can be seen as if the existing facilities are chosen between a given set of dimensional structures. We have characterized the whole set of optimal solutions of these problems and we have provided extensions to new models of location problems.

\section{References}

[1] Carrizosa E, Conde E, Fernández FR, Puerto J (1994) An axiomatic approach to the centdian criterion. Location Science 3:165-171

[2] Drezner Z (1995) Facility location: a survey of applications and methods. Springer-Verlag, New York

[3] Drezner Z, Hamacher HW (2002) Facility location: applications and theory. SpringerVerlag, Berlin Heidelberg

[4] Durier R, Michelot C (1985) Geometrical properties of the Fermat-Weber problem. European Journal of Operational Research 20:332-343

[5] Durier R (1995) The general one center location problem. Mathematics of Operations Research 20(2):400-414

[6] Elzinga J, Hearn DW (1972) Geometrical solutions for some minimax location problems. Transportation Science 6:379-394

[7] Fliege J (2000) Solving convex location problems with gauges in polynomial time. Studies in Locational Analysis 14:153-171

[8] Flury BA (1990) Principal points. Biometrica 77:33-41

[9] Hamacher HW, Nickel S (1998) Classification of location models. Location Science 6:229242

[10] Hiriart-Urruty JB, Lemaréchal C (1993) Convex analysis and minimization algorithms. Springer-Verlag, Berlin Heidelberg

[11] Johnson CR, Nylen P (1991) Monotonicity of norms. Linear Algebra Appl. 148:43-58

[12] Love RF, Morris JG, Wesolowsky GO (1988) Facilities location: models and methods. North-Holland, New York

[13] Megiddo N, Tamir A (1983) Finding least-distance lines. SIAM J. on Algebraic and Discrete Methods 4(2):207-211

[14] Morris JG, Norback JP (1980) A simple approach to linear facility location. Transportation Science 14:1-8

[15] Morris JG, Norback JP (1983) Linear facility location-solving extensions to the basic problem. European Journal of Operational Research 12:90-94 
[16] Robert JM, Toussaint GT (1990) Computational geometry and facility location. Proc. International Conf. on Operations Research and Management Science, Manila, The Philippines, B-1-B-19

[17] Rockafellar RT (1970) Convex Analysis. Princeton University Press, NJ

[18] Rodriguez-Chia A, Nickel S, Puerto J, Fernández FR (2000) A flexible approach to location problems. Mathematical Methods of Operations Research 51:69-89

[19] Schöbel A (1998) Locating least-distant lines in the plane. European Journal of Operational Research 106:152-159. Some properties of location problems with block and round norms. Operations Research 32(6):1309-1327

[20] Ward JE, Wendell RE (1985) Using block norms for location models. Operations Research 33:1074-1090

[21] Wesolowsky GO (1993) The Weber Problem: History and Perspectives. Location Science $1: 5-23$

[22] Witzgall CJ (1964) Optimal location of a central facility: Mathematical models and concepts. Tehnical Report, National Bureau of Standars Report 19:239-244 